\title{
A SECURITY DV-Hop Localization Algorithm Resist Spoofing Attack
}

\author{
Wanli ZHANG, Qixiang SONG \\ College of Information Engineering, \\ Suzhou University, Suzhou, China \\ yxiaoying2000@163.com; \\ zhangwanli0557@aliyun.com
}

\begin{abstract}
In order to reduce the node position error of DV-Hop algorithm in wireless sensor network, the artificial bee colony algorithm is introduced to design the DV-Hop algorithm. A new ABCDV-Hop (Artificial Bee Colony DV-Hop) algorithm is proposed in this paper. Based on the traditional DV-Hop algorithm, by using the minimum hops of nodes and position information of anchor nodes, the average distance per hop is solved by artificial bee colony algorithm to make it more close to the actual value. The simulation results show that compared with the traditional DV-Hop algorithm, the improved algorithm can effectively reduce the positioning error without increasing the node hardware overhead.
\end{abstract}

Keywords: DV-Hop algorithm; Artificial bee colony algorithm; The average distance per-hop

\section{Introduction}

Wireless sensor network has been widely used in industrial production, environmental monitoring, military reconnaissance and other fields [1]. Positioning technology of node is the basis of all kinds of application [2]. Wireless sensor network has characteristics of limited energy of the nodes, poor reliability, large scale and random distribution, the communication distance is limited and so on [3], so the positioning technology are researched more without ranging at this stage.

DV-Hop[4] positioning algorithm is the range free localization algorithm which is the most extensive research and application, but the precision of this algorithm is low. Aiming at this problem, the literature [5-10] has made some improvement on it. In view of the error which caused by average distance per- hop of anchor nodes, combining the theory of artificial bee colony algorithm, an improved DV-Hop algorithm based on artificial bee colony is proposed in this paper. The simulation results show that the improved algorithm without extra hardware and communication overhead, the positioning accuracy has obvious improvement compared with the original algorithm.

* Xiaoying Yang is the corresponding author. 


\section{Error Analysis of DV-Hop Algorithm}

The basic idea of DV-Hop positioning algorithm is that distance between unknown nodes and other nodes is expressed by product of the average distance per-hop between nodes in the network and the number of hops between nodes, and then uses three sided measurement or the maximum likelihood estimation method to obtain the location information of nodes. The positioning process is as follows:

(1) By flooding with controllable, each anchor node broadcasts packet $\left\{\mathrm{id}_{\mathrm{i}},\left(\mathrm{x}_{\mathrm{i}}, \mathrm{y}_{\mathrm{i}}\right)\right.$, hop $\left._{\mathrm{i}}\right\}$ which contains identification number, its coordinate position and hop to the network. Neighbor nodes record packet information ignoring packet from the same node with larger hops. Then packet's hops value plus 1 and forwarded to their neighbour nodes. By this stage, all the nodes obtain the minimum number of hops for each beacon node.

(2) The anchor nodes by using the Eq. (1) to calculate the average distance of each hop.

$$
H_{i}=\sum_{i \neq j} \sqrt{\left(x_{i}-x_{j}\right)^{2}+\left(y_{i}-y_{j}\right)^{2}} / \sum_{i \neq j} h_{i j}
$$

Where $\left(x_{i}, y_{i}\right),\left(x_{j}, y_{j}\right)$ are respectively coordinates of the node $i, j$, hop $p_{i j}$ as the minimum hop number between two nodes.

Then the $\mathrm{H}$ is broadcast to the network, the unknown node only down the first $\mathrm{H}$, and then the hop in the table is multiplied by $\mathrm{H}$ to obtain estimated distance of each anchor node.

(3) Using the three sided measurement or the maximum likelihood estimation method to calculate the position coordinates of unknown nodes.

Distance as long as within the communication radius $\mathrm{R}$, both near and far are recorded as 1 jump, as shown in Figure 1. Node A and node B, A and C despite the distance difference is very big, but both of them in the communication radius, and therefore distance between $\mathrm{A}$ and $\mathrm{B}$ and distance between $\mathrm{A}$ and $\mathrm{C}$ are recorded as 1 jump, so average distance per hop calculated through (1) have a big error. Resulting eventually localization error of nodes is large.

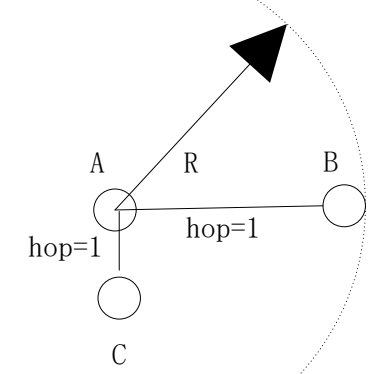

Figure 1. Schematic Diagram of Hop 


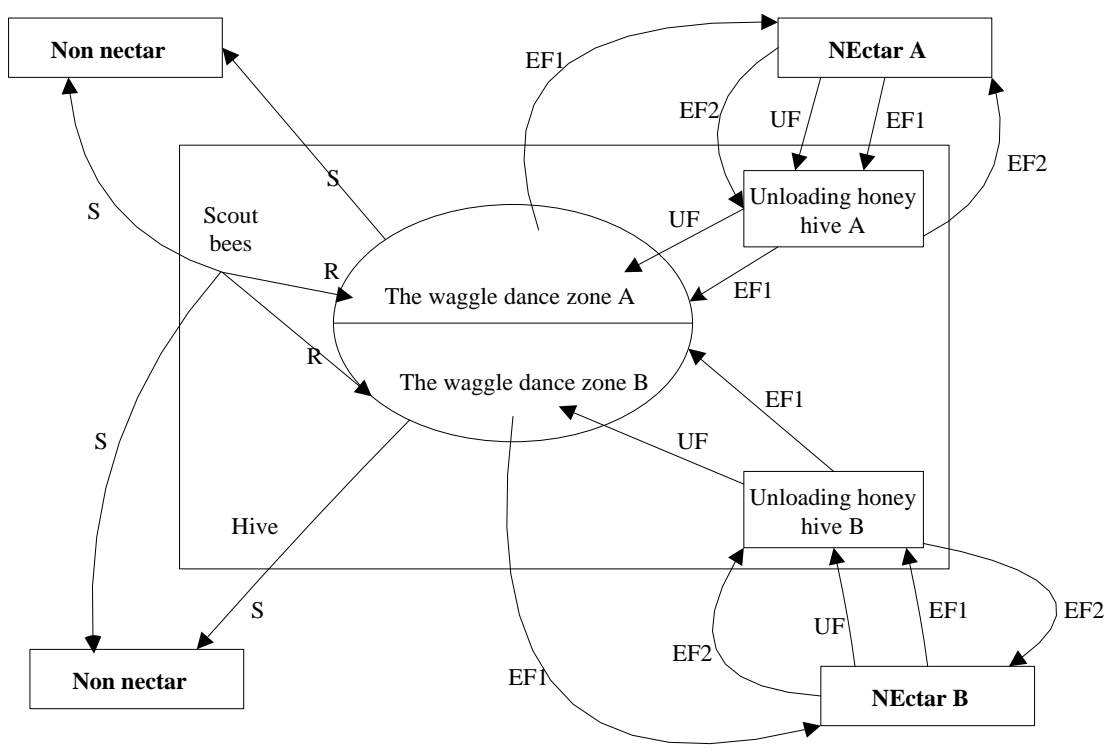

Figure 2. Mechanism of Bees Gathering Honey

\section{ABCDV-Hop Algorithm}

\subsection{The Principle of ABC Algorithm}

Artificial bee colony algorithm [11,12] (Artificial Bee Colony, ABC) is an optimization algorithm based on swarm intelligence by bees, through simulating the bees which have their own division of work intelligent honey and exchange honey source information to get the optimal solution. The algorithm is robustness, adaptability and can quickly find the optimal solution because of division of labor and cooperation mechanism.

In the algorithm, bees gather honey behavior mainly consists of 3 basic parts: honey, employed foragers (EF), unemployed foragers (UF). (1) Honey. Honey represents the various possible solutions. (2) Employed foragers. Employed foragers carry specific nectar source information which contains distance between honey and honeycomb, direction of nectar source, profitability of nectar. Employed foragers sharing this information with other bees by a waggle dance, according to a certain proportion, part to be leader bees. (3) Unemployed foragers. Unemployed foragers are divided into scout bees and follow bees. Scout bees search new nectar near hives. Follow bees wait the hive, look for nectar by sharing information of employed foragers. Mechanism of bees gathering honey is shown in Figure 1.

Assume there are two nectar sources which have been found: A and B. At the beginning, unemployed foragers have no information of nectar near the nest, so there are two choices: (a) unemployed foragers can be used as scout bees and they begin spontaneously search nectar near $r$ the nest (S. line in Figure 1). (b) Observation of the waggle dance to the other, it can be recruited and looking for nectar source according to the acquired information (R. line in Figure 1). After unemployed foragers find new nectar, 
they remember the position of the nectar and quickly begin to gather honey, at this time, unemployed foragers become employed bees.

The initial time, bees are scout bees without any prior knowledge. Random search to the food source, the scout bee return to dance area of the hive, according to the relative size of the food source of profitability, the scout bees can turn into any one of the above-mentioned bee.

\subsection{The ABC Algorithm is Used to Solve the Average Distance Per Hop}

From the analysis on the festival, the error of average distance per-hop is the main factor affecting the positioning accuracy of DV-Hop algorithm. Therefore, use artificial bee colony algorithm to calculate the optimal average distance per-hop, improve the accuracy of estimated average distance per-hop, thereby reducing the positioning error of DV-Hop algorithm.

Set $\mathrm{H}_{\mathrm{i}}$ be the average distance per-hop of the anchor nodei, according to the first stage of DV-Hop positioning algorithm, using the Eq. (2) to calculate the actual distance $d_{i j}$ between the anchor node $\mathrm{i}$ and anchor node $\mathrm{j}$, so estimation of the distance between two nodes is $\mathrm{H}_{\mathrm{i}} \times$ hop $_{\mathrm{ij}}$.

$$
d_{i j}=\sqrt{\left(x_{i}-x_{j}\right)^{2}+\left(y_{i}-y_{j}\right)^{2}}
$$

The error between the actual distance and estimated distance of anchor node $i$ and anchor node $\mathrm{j}$ are written as $\varepsilon$. The optimal $\mathrm{H}_{\mathrm{i}}$ should make $\varepsilon$ be minimum. Therefore the solution of $\mathrm{H}_{\mathrm{i}}$ is change into optimization problems of $\mathrm{H}_{\mathrm{i}}$. The sum of squares of errors as objective function $\mathrm{G}\left(\mathrm{H}_{\mathrm{i}}\right)$. As shown in Eq. (3), selecting an appropriate $\mathrm{H}$ makes the minimum function value.

$$
\mathrm{G}\left(\mathrm{H}_{\mathrm{i}}\right)=\min \sum_{\mathrm{i} \neq \mathrm{j}}\left(\mathrm{d}_{\mathrm{ij}}-\mathrm{H}_{\mathrm{i}} \times \mathrm{hop}_{\mathrm{ij}}\right)^{2}
$$

In the process of artificial bee colony algorithm, to solve the optimal solution is to seek the profit degree food source. The corresponding relationship between to solve the optimal $\mathrm{H}_{\mathrm{i}}$ problem and bees gather honey behavior is shown in Table 1 .

Table 1: Relationship between $H_{i}$ Optimization and

\begin{tabular}{|c|c|}
\hline bees gather honey behavior & $H_{i}$ optimization problem \\
\hline $\begin{array}{c}\text { Nectar source location } \\
\text { Honey yield size } \\
\text { Speed of Seeking and collecting } \\
\text { nectar } \\
\text { High yield of }\end{array}$ & $\begin{array}{c}\text { All possible solutions of } H_{i} \\
\text { The quality of the solution } H_{i} \\
\text { The optimization speed of the solution } H_{i} \\
\text { The optimal solution } H_{i}\end{array}$ \\
\hline
\end{tabular}
Bees Gather Honey Behavior

When distance between two points is far to a certain extent, two points will no can communicate with each other [13]. Therefore, the maximum distance between two points 
should be similar to the communication radius of sensor node. So the value of $\mathrm{H}_{\mathrm{i}}$ should satisfy Equation (4).

$$
0 \leq \mathrm{H}_{\mathrm{i}} \leq \mathrm{R}
$$

Because the problem is solving the minimal value of the objective function, so the fitness function is selected as

$$
\text { fit }_{\mathrm{i}}=\frac{1}{\alpha+\beta \mathrm{G}\left(\mathrm{H}_{\mathrm{i}}\right)}
$$

$\alpha$ and $\beta$ Said fitness function coefficient. Adaptive value is greater, solution $\mathrm{H}$ value is more close to the actual.

Based on the above analysis, steps of using artificial bee colony algorithm to find out the optimal solution of $\mathrm{H}$ is as follows:

Step1 : initialize population. $\mathrm{N}_{\mathrm{s}}$ feasible solution are randomly generated by Equation (6), to form the initial population. The first 50\% are as leader bees, after 50\% are as follow bees.

$$
\mathrm{H}_{\mathrm{i}}=\mathrm{H}_{\mathrm{imin}}+\operatorname{rand}(0,1)\left(\mathrm{H}_{\mathrm{imax}}-\mathrm{H}_{\mathrm{imin}}\right)
$$

Among, $\quad \mathrm{i} \in\left(1,2, \cdots, \mathrm{N}_{\mathrm{S}}\right), \mathrm{H}_{\mathrm{imin}}$ and $\mathrm{H}_{\mathrm{imax}}$ respectively express the minimum and maximum values of ith component of $\mathrm{H}$. According to formula (4), $\mathrm{H}_{\mathrm{imin}}=0$, $\mathrm{H}_{\mathrm{imax}}=\mathrm{R}$.

Step 2: Calculate fitness value. Calculate fitness value of each H through Eq.(5).

Step 3 : Bees record the optimal value so far, and expand the neighborhood search in the current nearby honey. A new $\mathrm{H}_{\mathrm{i}}^{\prime}$ is generated by Eq. (7) to instead $\mathrm{H}_{\mathrm{i}}$.

$$
\mathrm{H}_{\mathrm{i}}^{\prime}=\mathrm{H}_{\mathrm{i}}+\emptyset_{\mathrm{i}}\left(\mathrm{H}_{\mathrm{i}}-\mathrm{H}_{\mathrm{k}}\right)
$$

Among, $\quad \mathrm{i} \in\left(1,2, \cdots, \mathrm{N}_{\mathrm{c}}\right), \mathrm{k} \in\left(1,2, \cdots, \mathrm{N}_{\mathrm{c}}\right), \quad \mathrm{N}_{\mathrm{c}}=\frac{1}{2} \mathrm{~N}_{\mathrm{s}}, \mathrm{k}$ is the random generation and $\mathrm{k} \neq \mathrm{i}, \quad \emptyset_{\mathrm{i}} \in[-1,1]$.

Step 4 : Calculate yield of the new generation individual of $\mathrm{H}_{\mathrm{i}}^{\prime}$ by Equation (5).

Step $5:$ Bees use greedy principle to the optimal solution $\mathrm{H}_{\mathrm{i}}$ in memory to the solution $\mathrm{H}_{\mathrm{i}}^{\prime}$ which is obtained by neighborhood search. When the search optimal solution is better than the memory of the solution, replace the memory solution; on the other hand, remain unchanged.

Step 6: Generate scout bees. Through step 5, bees which the fitness value does not change and to limit the number of individual limit, according to Eq. (8) is converted to scout bees.

$$
\mathrm{H}_{\mathrm{zi}}=2\left(\mathrm{H}_{\mathrm{imax}}-\mathrm{H}_{\mathrm{imin}}\right)(0.5-\operatorname{rand}(0,1))
$$

Step 7: Return to step 2 is it does not satisfy the convergence condition, until the end of the cycle, output the optimal solution of $\mathrm{H}$. 


\subsection{Realization ABCDV-Hop algorithm}

The flow chart of improved algorithm with $\mathrm{ABC}$ algorithm in DV-Hopis shown in Figure 3.

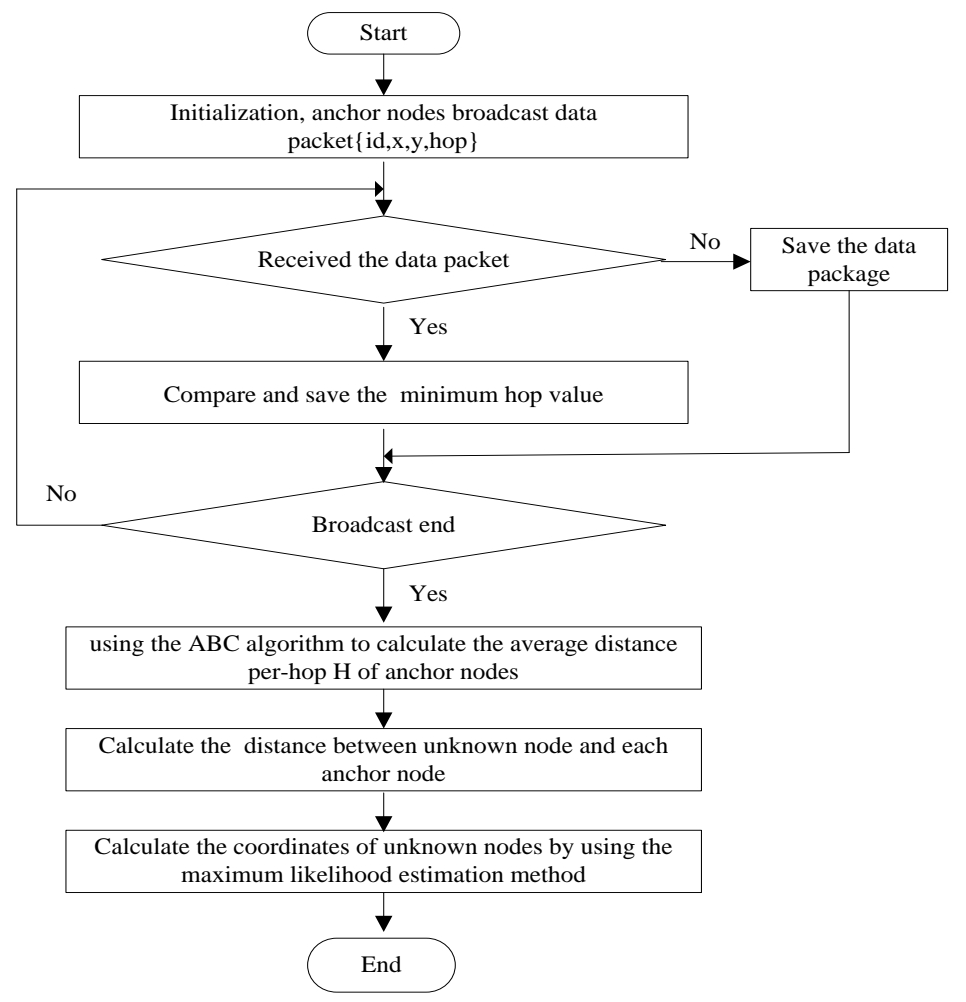

\section{Figure 3. ABCDV-Hop Algorithm Flow Chart}

\section{The Simulation Experiment}

In order to compare the performance of ABCVDV-Hop algorithm and the traditional DV-Hop algorithm, simulation experiments are carried out on the platform of MATLAB 7. The precision of traditional algorithm and the improved algorithm were studied under different proportion of anchor node, different number of nodes and different communication radius.

\subsection{The Simulation Environment and Parameter Setting}

Set all nodes are randomly distributed in a square area of $100 \mathrm{~m} * 100 \mathrm{~m}$, beacon nodes are selected randomly from the area. All nodes have same radius $R=10 \mathrm{~m}$, the number of colony is $N_{s}=50, \alpha=\beta=1$. The number of cycles for colony is 200 , limit=20. In order to eliminate instability of errors caused by node random distribution, in the same network environment simulation 100 times of experiments is taken respectively and takes the average value.

The normalized localization error is a measure of the localization algorithm accuracy index. Defined as: 


$$
\text { error }=\left[\sum_{j=1}^{\mathrm{M}} \sum_{\mathrm{i}=1}^{\mathrm{N}}\left(\sqrt{\left(\mathrm{x}_{\mathrm{i}}^{\prime}-\mathrm{x}_{\mathrm{i}}\right)^{2}+\left(\mathrm{y}_{\mathrm{i}}^{\prime}-\mathrm{y}_{\mathrm{i}}\right)^{2}}\right)^{2}\right] / \mathrm{MNR}
$$

In the Equation (9), M, N, R respectively is the number of simulation, the unknown nodes and node communication radius. $\left(\mathrm{x}_{\mathrm{i}}^{\prime}, \mathrm{y}_{\mathrm{i}}^{\prime}\right),\left(\mathrm{x}_{\mathrm{i}}, \mathrm{y}_{\mathrm{i}}\right)$ were estimated coordinates of nodes and the real coordinates of nodes.

\subsection{Positioning Accuracy Analysis}

4.2.1 The Relationship Between Proportion of Anchor Node and Positioning Accuracy: The total number of nodes is set to 200; the anchor node proportion increases from $10 \%$ to $50 \%$, comparison of the normalized localization error of the ABCVDV-Hop algorithm and traditional DV-Hop algorithm are shown in Figure 4.

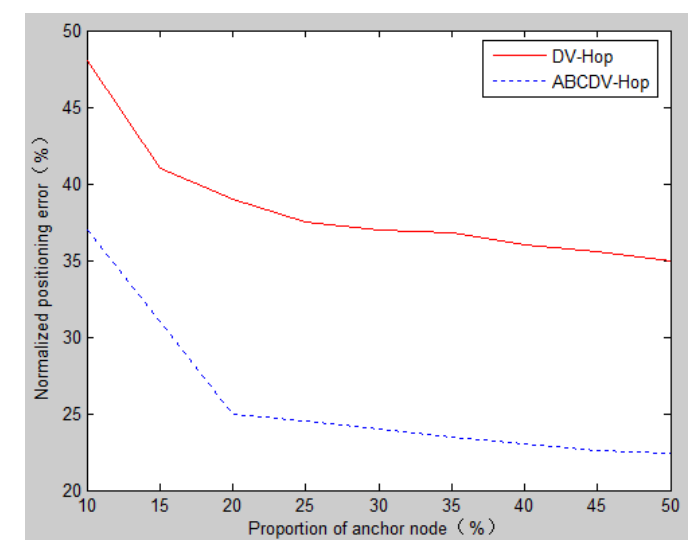

\section{Figure 4. Relation of Anchor Node Proportion and Normalized Localization Error}

As can be seen from Figure 4, normalized localization error decreased and stabilized with the increase in the proportion of beacon nodes. In the same proportion of anchor node, because the average distance per- hop is optimized in the ABCVDV-Hop algorithm, the average distance per- hop which is gotten is closer to the actual value, therefore the positioning accuracy is significantly higher than that of the positioning accuracy of the traditional DV-Hop algorithm. ABCVDV-Hop algorithm is better than traditional DV-Hop algorithm in average positioning accuracy, which is improved about $15.5 \%$.

4.2.2. The Relationship Between Node Number and the Positioning Accuracy: The fixed anchor node proportion is $10 \%$, the communication radius keep unchanged, the number of nodes from 100 to 500, comparison results of the normalized localization error of ABCVDV-Hop algorithm and the traditional DV-Hop algorithm is shown in Figure 5. 


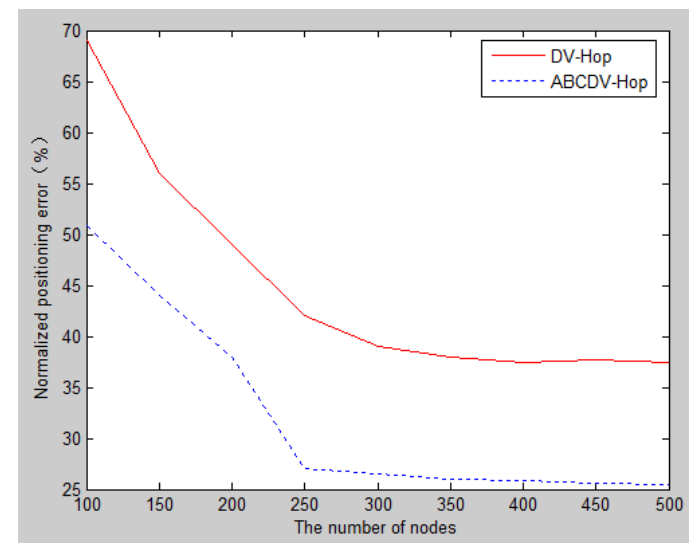

\section{Figure 5. Relation between Node Number and Normalized Localization Error}

As can be seen from Figure 5, the normalized localization error of two algorithms show a decreasing trend with the increase of the total number of nodes, and are stabilizing after nodes in the total reaching 300. The node data of the network in the region reach saturation at this point, if increasing the number of nodes; the normalized localization error will not be decreased significantly. When the number of nodes is same, ABCVDV-Hop algorithm is better than traditional DV-Hop algorithm in average positioning accuracy, which is improved about $12.7 \%$.

\subsubsection{The Relationship between Communication Radius and Location Accuracy:} The number of nodes is 100 , the proportion of anchor node is $30 \%$. Comparison results of the normalized localization error of ABCVDV-Hop algorithm and the traditional DV-Hop algorithm is shown in Figure 6 , in different communication radius.

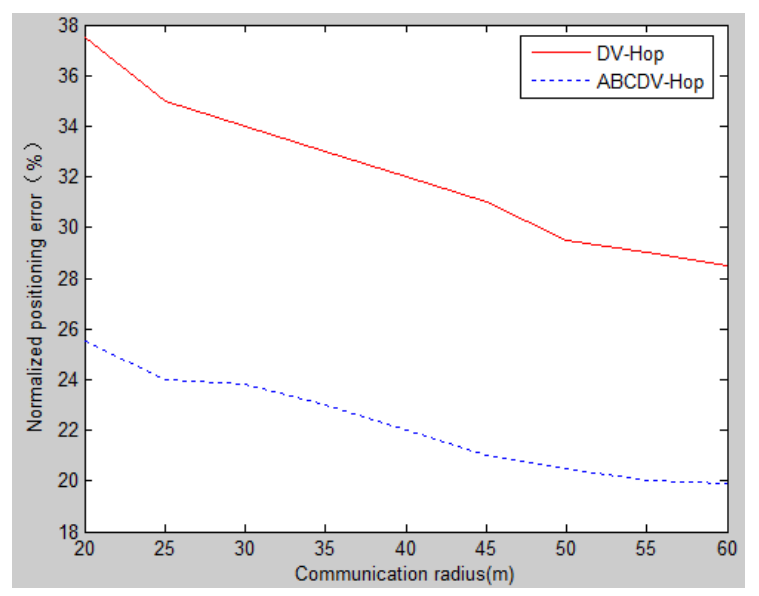

Figure 6. The Relationship between Communication Radius and the Normalized Localization Error

As can be seen from Figure 6, node localization error of the two algorithms has decreased with the increase of radius of communication. But the effect by the minimum 
communication radius of ABCVDV-Hop algorithm is smaller, because the improved algorithm can optimize the average distance per hop of anchor nodes. The error of average distance per-hop is not obviously increased with the radius increasing. The average positioning accuracy of ABCVDV-Hop algorithm is better than that of traditional DV-Hop algorithm, which is improved about $14.3 \%$.

\subsection{Analysis of the Complexity of the Algorithm}

The total number of nodes in the network is set to $\mathrm{N}$, the beacon node number is set to A. The communication radius is set to $20 \mathrm{~m}$; the data of anchor node proportion is $15 \%$. The communication overhead and the complexity of the algorithm are shown in table 2 . As ABCVDV-Hop algorithm just uses ABC algorithm to calculate the average distance per-hop of anchor nodes, therefore, the algorithm slightly increases the amount of computation, did not change the transmission path of data packets and do not increase the number of packets, so the communication overhead does not increase. As can be seen from table 2, compared with the traditional DV-Hop algorithm, the improved algorithm obtain higher positioning accuracy with a small amount of calculation but without increase the communication overhead.

Table 2: Comparison of Performance of the Two Algorithms

\begin{tabular}{lcr}
\hline Performance ABCDV-Hop algorithm & DV-Hop algorithm \\
\hline Positioning accuracy & $80 \%$, high & $66 \%$, low \\
Communication overhead & $2 * \mathrm{~N}^{*} \mathrm{~A}$ & $2 * \mathrm{~N}^{*} \mathrm{~A}$ \\
Computation complexity & big & small \\
Hardware complexity & $\begin{array}{c}\text { No additional hardware } \\
\text { hardware }\end{array}$ & No additional \\
\hline
\end{tabular}

\section{Conclusion}

According to the positioning error caused by average distance per-hop in DV-Hop, based on analyzing the principle of artificial bee colony algorithm, artificial bee colony algorithm is applied to DV-Hop positioning algorithm to improve the calculation method of average distance per hop of anchor nodes. The algorithm has less control parameters, the search accuracy is high and the operation is stable. It can quickly find the optimal solution by determining the fitness function. ABCVDV-Hop algorithm improves the accuracy of average distance per hop and reduces the positioning error. Simulation results show that in situation of different anchor node proportion, different number of nodes and different communication radius, ABCVDV-Hop algorithm significantly improves the positioning accuracy without increasing the communication overhead and additional hardware. 


\section{Acknowledgements}

The study of our work is supported in part by Young Talents Fund Project in Anhui Province of China (No. 2013SQRL083ZD), Anhui University Provincial Natural Science Research Project (No.KJ2014A247) and University student's Innovative Training Project(201310379019).

\section{Reference}

[1] Z. Yan. "Study of Wireless Sensor Network Localization Algorithm Based on RSSI", Computer Science, vol. 36, no. 4 (2009).

[2] L. Yunfei, J. Ming, L. Ke, et al. "Improved DV-Hop localization algorithm in Wireless Sensor Network", Computer Engineering and Applications, vol. 50, no. 3 (2014).

[3] G. Yu, L. Jing, X. Bo, et al. "Wireless sensor network node localization based on shuffled frog leaping algorithm", Computer Engineering and Applications, vol. 48, no. 20, (2012).

[4] D. Niculescu , B. Nath . "DV based positioning in ad hoc networks", Journal of Telecommunication Systems, $22,1 / 4(\mathbf{2 0 0 3})$.

[5] W. Jiangtao, F. Xuemin, W. Xijun., “ Improved DV-Hop Location Algorithm Based on Hop Correction”, Chinese journal of sensor and actuator, vol. 27, no. 1 (2014).

[6] F. Jiang, Z. Qiang, W. Chun-chun, "Research on Improved DV-Hop Localization Algorithm", Computer Engineering, vol. 38, no. 19, (2012).

[7] W. Xin-sheng, Z. Yan-jing, L. Ha-i tao. "Improved Study Based on DV-Hop Localization Algorithm", Computer Science, vol. 38, no. 2 (2012).

[8] L. Qingming, B. Mengliang, Z. Wei. "A kind of improved DV-Hop Algorithm", Proc. of the 2nd International Conference on Intelligent Control and Information Processing. IEEE Press,(2011), Harbin, China

[9] L. Wenwen, Z. Wuneng. "Genetic algorithm base localization algorithm for wireless sensor network", Proc. of 2011 Seventh International Conference on Natural Computation, IEEE Press ,(2011), Shanghai, China

[10] L. Mu-dong, X. Wei, G. Long. Improvement of DV-Hop Localization Based on Artificial Bee Colony Algorithm[J], Computer Science, vol. 40, no. 1, (2013).

[11] D. KARABOGA. "An Idea Based On Honey Bee Swarm for Numerical Optimization", Turkey: Erciyes University, (2005).

[12] B. BASTURK, D. KARABOGA. "ArtificialBee Colony(ABC) Optimization Algorithm for Solving Constrained Optimization Problems", Foundations of Fuzzy Logic and SoftComputing, vol. 45 no. ,29 (2007).

[13] W. Shanshan, Y. Jianping,, C. Zhiping, and Z. Guomin, “A RSSI-Based Self-Localization Algorithm for Wireless Sensor Networks", Journal of Computer Research and Development, vol. 45, no. 1, (2008).
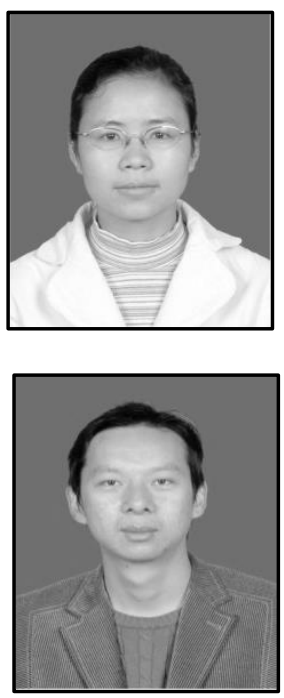

\section{Authors}

Xiaoying Yang, she was born in 1983. She received the master degree of computer technology from Guilin University of Electronic Technology in 2010. She is a lecturer at College of Information Engineering, Suzhou University, China. She research interests include Wireless Sensor Networks and database system.

Wanli Zhang, he was born in 1977. He received the master degree of computer technology from Anhui University in 2009. He is a lecturer at College of Information Engineering, Suzhou University, China. He research interests include Internet of Things and computer network. 